\title{
Modeling and Testing of a Kite-Powered Water Pump
}

David J. Olinger ${ }^{1}$, Alireza Mahdavi Nejad', Jitendra S Goela², Gretar Tryggvason³

${ }^{1}$ Mechanical Engineering Department, Worcester Polytechnic Institute, Worcester, MA,

${ }^{2}$ The Dow Chemical Company, Marlboro, MA

${ }^{3}$ Department of Aerospace and Mechanical Engineering, University of Notre Dame, Notre Dame, IN

This presentation summarizes recent work at Worcester Polytechnic Institute (WPI) to model, design, fabricate, and test a low-cost kite-powered water pump. The system is designed to be used in developing nations to alleviate water shortages. It uses a kite and tether that transmits the generated aerodynamic forces to a rocking arm, and through a mechanical linkage to a displacement (or lift) pump on the ground. Dynamic equations were developed for the kite, a flexible tether with applied lift, drag, and weight forces, the rocking arm, mechanical linkage and pump.

A steady-state analysis of the kite aerodynamics was incorporated into the dynamic equations of the kite-power system. The governing equations were solved numerically to assess how performance parameters of the system such as water pumping rate, tether profile and tension, and kite motion varied with tether length and diameter and wind speed. The results showed that for a kite area of 8 square meters and wind speeds of 6 meters per second, the operation of a kite powered water pump is feasible with a maximum water pumping rate of 8000 liters/day. This pumping rate would provide water for about 400 people in a developing nation. Design limits, above which system pumping rates were negatively affected, on tether length and diameter were determined from the water pump simulations to be about $500-\mathrm{m}$ and $3-\mathrm{mm}$, respectively, Ongoing efforts to build and test a working kite-powered water pump at WPI will be presented.

We will report on recent successful field tests of the system in spring and summer 2013. We will also summarize other work on airborne wind energy at WPI including early efforts to develop a Navier-Stokes based simulation of the power and retraction phases of a pumping cycle kite power system. In these simulations wind flow over a two-dimensional flexible kite is modeled, and kite motion is predicted.

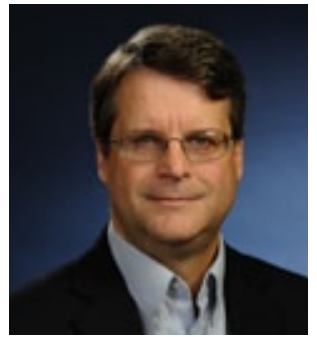

David J. Olinger

Worcester Polytechnic Institute Mechanical Engineering Department

100 Institute Road Worcester, MA 01609 USA

olinger@wpi.edu www.wpi.edu 